\title{
Recovery of the High-Acceleration Vestibulo-ocular Reflex After Vestibular Neuritis
}

\author{
A. Palla and D. Straumann \\ Neurology Department, Zurich University Hospital, Zurich, Switzerland
}

Received: 9 September 2003; Accepted: 23 July 2004; Online publication: 18 October 2004

\section{ABSTRACT}

Vestibular neuritis (VN) usually leads to a sudden gain asymmetry of the high-acceleration horizontal vestibulo-ocular reflex (VOR). We asked whether this asymmetry decreases over time indicating peripheral recovery and/or central compensation. The horizontal VOR during rapid rotational head impulses to both sides was recorded with search coils in 37 patients at different time periods (1-240 weeks) after the onset of VN. In ten patients, sequential measurements were performed. Gains of the VOR during head impulses toward the ipsilesional side significantly increased after the initial drop (average gains: < 1 week: 0.35 ; $1-4$ weeks: 0.33 ; $4-40$ weeks: 0.55 ; 40 240 weeks: 0.50 ). Gains on the contralesional side, however, were only slightly reduced and showed no significant change. We conclude that, in contrast to patients after hemilabyrinthectomy or unilateral vestibular neurectomy, the ocular response to ipsilesional rotations in patients after $\mathrm{VN}$ improves over time. This finding suggests that ipsilesional recovery is peripheral or, if central, depends on spared peripheral function. The physiology of linear and nonlinear VOR pathways predicts a considerable gain reduction for contralesional head impulses if central compensation mechanisms are not engaged. Thus, the relatively preserved gain on the contralesional side can be explained only by central "upregulation". Apparently, for high accelerations of the head, effective central compensation after $\mathrm{VN}$ does not aim to balance the gains of the VOR but tries to boost the contralesional gain close to normal.

Correspondence to: A. Palla - Neurology Department - Zurich University Hospital - Frauenklinikstrasse 26 - CH-8091 Zurich, Switzerland. Telephone: +41-1-255-5500; Fax: +41-1-255-4507; email: antpalla@access.unizh.ch
Keywords: neuro-otology, central compensation, VOR upregulation, head-impulse test, Ewald's second law, VOR pathways

\section{INTRODUCTION}

Vestibular neuritis (VN) is a sudden unilateral deficit of the peripheral vestibular organ (labyrinth or nerve) without auditory symptoms in otherwise healthy persons and leads to both static and dynamic vestibular imbalances. Recent studies have suggested a viral etiology of VN (Schuknecht and Kitamura 1981), most likely a reactivation of latent herpes simplex virus type 1 (Furuta et al. 1993; Schulz et al. 1998; Arbusow et al. 1999, 2000).

Signs and symptoms of VN due to static vestibular imbalance, such as postural instability, sensation of rotation, and spontaneous horizontal nystagmus, usually disappear within one week (Brandt 2001). Recovery from dynamic vestibular imbalance after VN is frequency-dependent (Paige 1989). When tested with low accelerations used in clinical turntable testing or caloric irrigation, the gains of the vestibuloocular reflex (VOR) become symmetrical within several weeks (Brantberg and Magnusson 1990; Imate and Sekitani 1993; Allum and Ledin 1999). When tested with high accelerations generated by Halmagyi-Curthoys head impulses (Halmagyi and Curthoys 1988), however, the horizontal VOR often remains asymmetrical, even after many years (Schmid-Priscoveanu et al. 1999, 2001; Aw et al. 2001).

Patients with permanent unilateral vestibular loss due to vestibular neurectomy also show gain asymmetries of the horizontal VOR evoked by head im- 
pulses. In these patients, the magnitude of asymmetry does not change for many years (Halmagyi et al. 1990; Aw et al. 1996, 2001). Thus, a unilaterally absent peripheral function seems to be associated with a persistent gain asymmetry of the high-acceleration VOR. Conversely, an ipsilesional gain increase would indicate either incremental peripheral recovery or progressively more effective central compensation making use of the spared ipsilesional peripheral vestibular input. We asked whether, in fact, the gain asymmetry of the high-acceleration VOR after VN would decrease over time as a result of residual peripheral vestibular function.

After VN, the gain of the VOR evoked by contralateral head impulses is, on average, slightly below normal (Schmid-Priscoveanu et al. 1999; Aw et al. 2001). The same small contralesional gain reduction is also found in patients after unilateral vestibular neurectomy (Halmagyi et al. 1990; Aw et al. 1996, 2001). It is still unclear to what extent the unilateral peripheral deficit and central compensation mechanisms determine the gain on the contralesional side. Using a realistic model of the VOR (Lasker et al. 2000), we asked whether the contralateral gain reduction of the high-acceleration VOR in patients after VN can be explained by a unilateral deficit with or without central compensation mechanisms alone or whether, in some cases, an additional involvement of the contralateral labyrinth or vestibular nerve is likely.

\section{MATERIAL AND METHODS}

\section{Subjects}

Thirty-seven patients (21 male, 27-83 years old) diagnosed with vestibular deficit due to clinically suspected vestibular neuritis (VN) were included in the study. The patients were partitioned into four groups according to the time passed from the onset of the vestibular deficit to the date of the examination (group I: 1 day-1 week; group II: 1-4 weeks; group III: 4-40 weeks; group IV: 40-240 weeks). These intervals represent an approximate logarithmic distribution. Ten patients were assigned to two different groups, since they could be tested twice at different time periods after the onset of the deficit. The total number of testing sessions was 47 (group I: 6; group II: 6; group III: 11; group IV: 24). The comparison group consisted of 11 healthy human subjects (5 male, 25-39 years old).

Informed consent of patients and healthy subjects was obtained after full explanation of the experimental procedure. The protocol was approved by a local ethics committee and was in accordance with the ethical standards laid down in the 1964 Declara- tion of Helsinki for research involving human subjects.

\section{Quantitative head impulse testing}

Eye and head movements were recorded in a magnetic frame (Remmel-type system, modified by A. Lasker, Baltimore, MD, USA) using dual search coils (Skalar Instruments, Delft, Netherlands) that were calibrated before each session (for details see Straumann and Zee 1995). One search coil was placed on the right eye around the cornea (after anesthetizing the conjunctiva with oxybuprocaine $0.4 \%$ ), the other was tightly fixed on the forehead with adhesive tape. Voltages were sampled with 16 bits at $1000 \mathrm{~Hz}$ and stored on the hard disk of a computer.

During experiments, subjects were seated inside the magnetic coil frame (side length $=1.4 \mathrm{~m}$ ). Care was taken to position the center of the interpupillary line in the center of the magnetic frame. Horizontal head impulses (amplitude $=20^{\circ}-40^{\circ}$; duration $=$ $150-200 \mathrm{~ms}$; peak velocity $=\sim 300^{\circ} / \mathrm{s}$; peak acceleration $=\sim 10,000^{\circ} / \mathrm{s}^{2}$ ) were applied by the investigator standing behind the subject. The directions of head impulses were pseudorandomly intermingled; four to six head rotations were applied to each side. Subjects were instructed to always fix upon a light dot $1.24 \mathrm{~m}$ straight ahead.

\section{Data analysis}

Digitized signals were processed using interactive programs written in MATLAB (version 6.1). Since it is not known whether $\mathrm{VN}$ affects only the gain or, in addition, the latency of the vestibulo-ocular reflex, we used an analytical method that implicitly discards the effect of latency on the gain value. ${ }^{1}$ This was achieved by plotting head-in-space against eye-in-space. The gain of the vestibulo-ocular reflex, $g$, was computed using the formula:

$$
g=\frac{\Delta e_{s}\left[h_{0} ; h_{1}\right]}{h_{1}-h_{0}}
$$

where $h_{0}$ and $h_{1}$ are head-in-space positions, and $\Delta e$ is the difference between eye-in-space positions at $h_{0}=3^{\circ}$ and $h_{1}=7^{\circ}$. Traces of head impulses traversing the position interval $\left[h_{0} ; h_{1}\right]$ were relatively straight. In every trial, this position interval was traversed after the moment of peak head acceleration and before the moment of peak head velocity.

\footnotetext{
${ }^{1}$ Increased latency leads to a false reduction of gain, if determined at peak head velocity.
} 
FIG. 1. Examples of horizontal head impulse tests in a healthy subject (A) and a patient two weeks (B) and two months (C) after a sudden vestibular asymmetry. If the vestibulo-ocular reflex (VOR) were perfectly compensatory, traces would be parallel to the abscissa (head-in-space axis); if the vestibulo-ocular reflex were absent, traces would move on a $45^{\circ}$ slope. $G_{R}$ : median gain value for head impulses to the right; $G_{\mathrm{L}}$ : median gain value for head impulses to the left. Traces are clipped beyond $10^{\circ}$ eccentricity of head-in-space. Dashed vertical lines indicate intervals used to determine the gains (see Methods).

Median gains during head impulses to the right $\left(G_{\mathrm{R}}\right)$ and left $\left(G_{\mathrm{L}}\right)$ side were calculated. For convenience, the gains of patients and healthy subjects were mirrored, if the gain on the right side was higher than the gain on the left side. Thus, in the analysis of the patients' data, the right side is always ipsilesional and the left side contralesional.

\section{RESULTS}

Figure 1 contains three plots of head and eye movements during horizontal head impulses to both sides in a typical healthy subject (Fig. 1A) and a typical patient (Fig. 1B, C). In the healthy subject (Fig. 1A), gains were symmetric but not completely compensatory. In the patient, the gain for ipsilesional head impulses was markedly reduced two weeks after the onset of vestibular neuritis (Fig. 1B), while the contralesional gain was somewhat yet not significantly lower than gains in healthy subjects. The same patient was tested two months later (Fig. 1C). The ipsilesional gain had increased, and the contralesional gain was only slightly above the previous value. Hence, the difference between both gains became smaller.

Figure 2 plots average gain values of each group of patients during head impulses toward the ipsilesional (Fig. 2A) and the contralesional (Fig. 2B) side, as well as the difference between the two sides (Fig. 2C). During head impulses toward the ipsilesional side (Fig. 2A), average gain values were around 0.35 during the first week (group I) and subsequent three weeks (group II), but they then rose to a level of around 0.50 (groups III and IV). Gains measured during head impulses toward the contralesional side (Fig. 2B) were, on average, somewhat below normal values. The decrease, however, was not significant and also showed no significant change over time. Differences between ipsi- and contralesional gains (Fig. 2C) were around 0.35 during the first four weeks (groups I and II) and then decreased to around 0.2 (groups III and IV).

Ten patients could be tested twice at different time intervals after the onset of the vestibular deficit. Figure 3 illustrates the ipsi- and contralesional gain val-
A

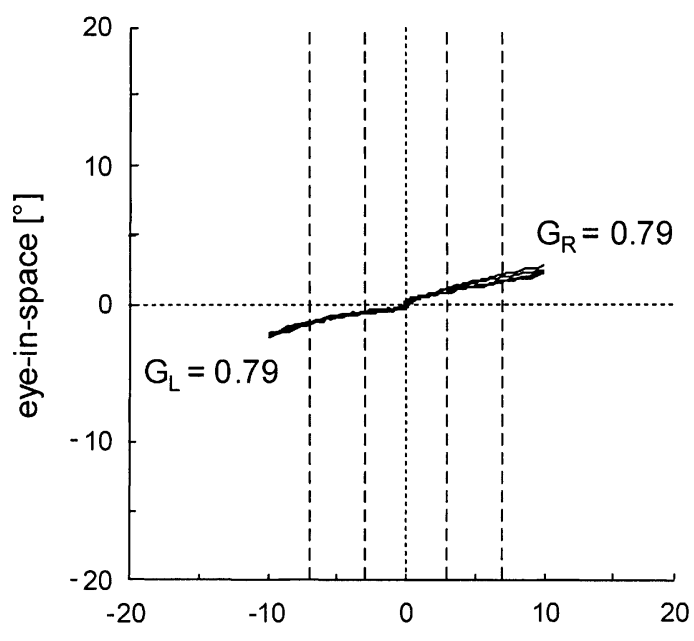

B

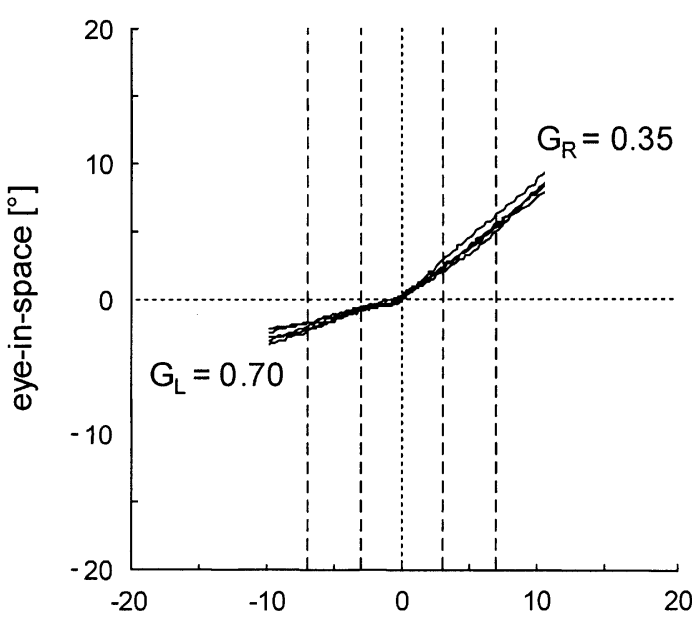

C

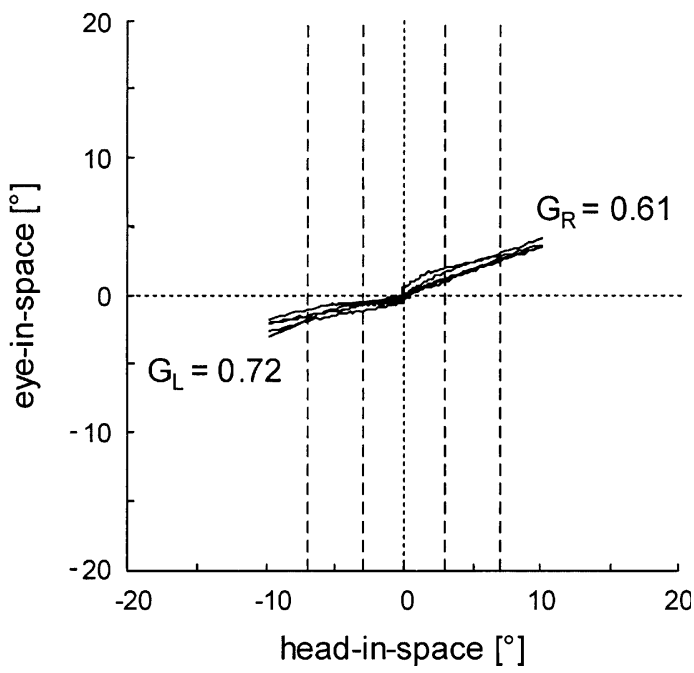

ues of these patients in the same format as in Figure 2. Again, there was a clear tendency of the ipsilesional gain to improve over time (Fig. 3A), while the contralesional gain was slightly but not significantly below normal and stayed at that level (Fig. 3B). 
A

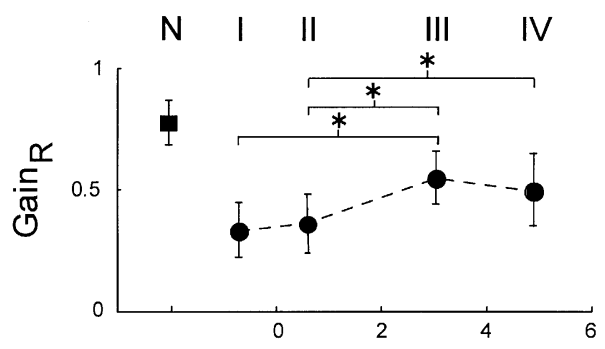

B

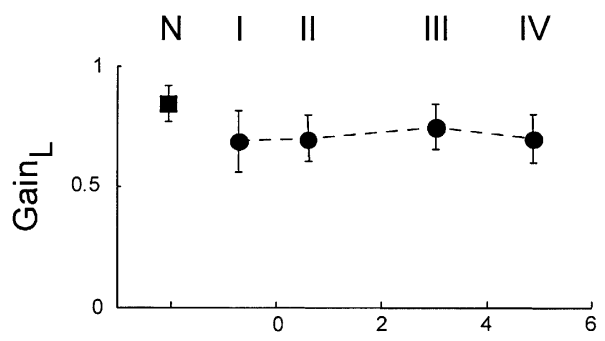

C

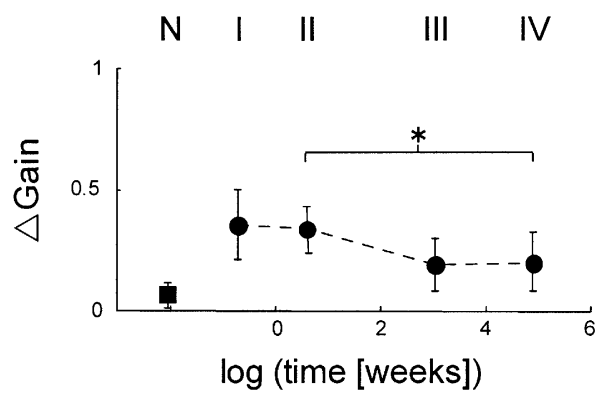

$* p<0.05$

FIG. 2. Average gain values (error bars: $\pm 1 \mathrm{SD}$ ) of the four groups of patients (I, II, III, IV) and the group of healthy subjects (N). The abscissa indicates logarithmic time, except for the group of healthy subjects. In all panels, averages in the four groups of patients were significantly different from averages in the group of healthy subjects $(p<0.05$ in the unpaired two-tailed $t$-test). Stars indicate significant differences of averages between patient groups. A. Head impulses toward the right side. B. Head impulses toward the left side. C. Gain differences (left minus right gain). Note that the gains were mirrored in subjects in whom the gain on the right side was higher than on the left side. Thus, the right side was always the weaker side and, as a result, the gain differences were always positive (see Methods).

As a consequence, the asymmetry between ipsi- and contralesional gains decreased as a function of time (Fig. 3C).

So far, we have shown that the gain of the VOR during head impulses toward the ipsilesional side partly recovered after the onset of the deficit. To interpret the gain values during head impulses toward the contralesional side, we compared our data with the predictions of a recent model by Lasker et al. (2000), which is based on ocular responses of hemilabyrinthectomized squirrel monkeys during highfrequency, high-acceleration rotations. In these ani-
A

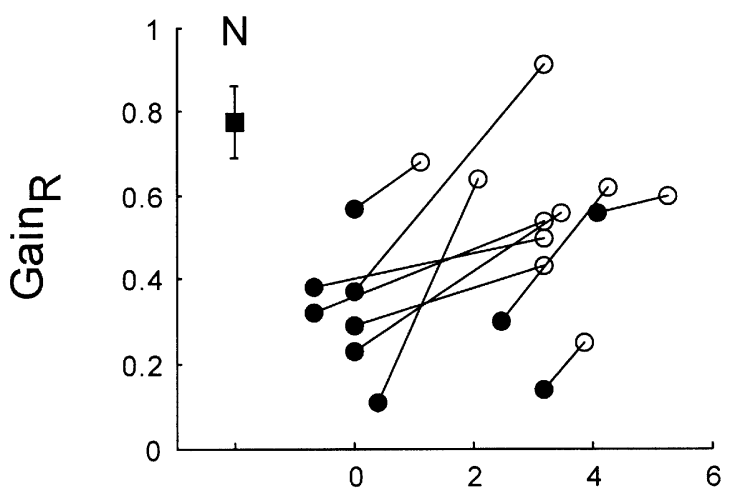

B
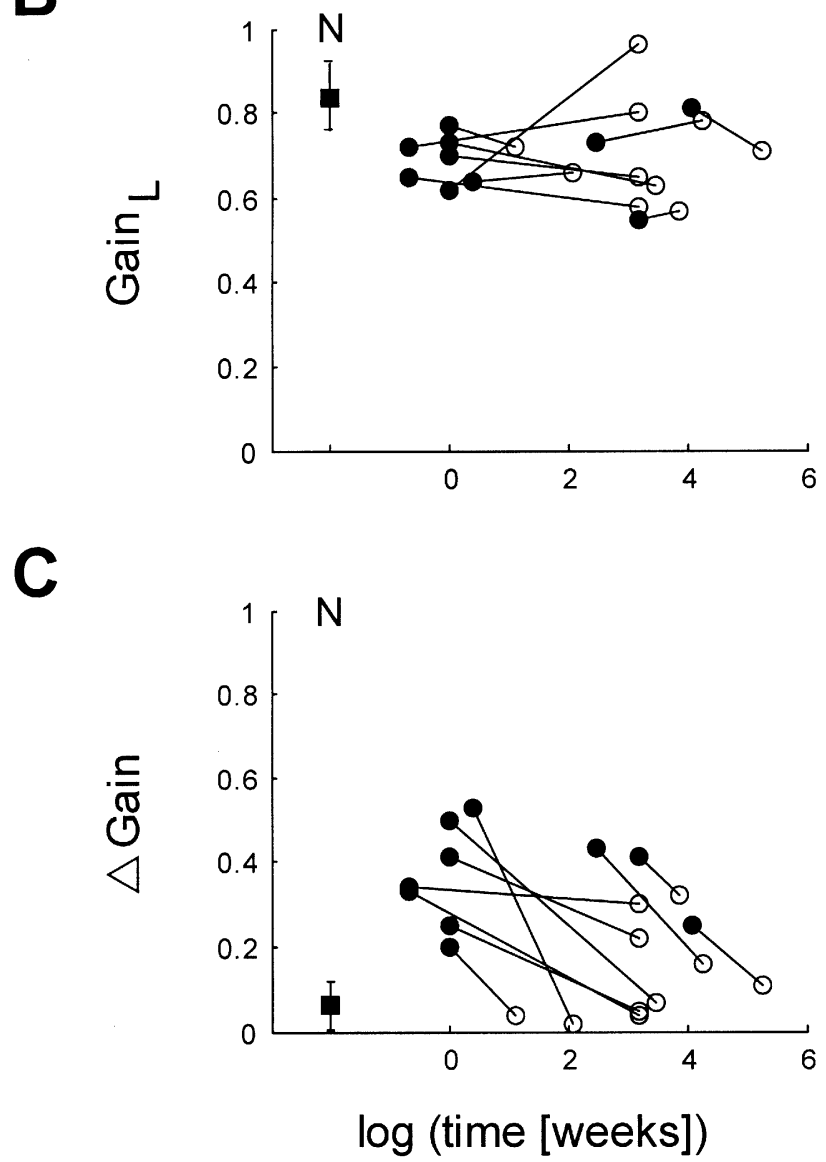

FIG. 3. Individual gain values in the ten patients who could be tested twice at different time periods after the onset of vestibular neuritis. Panels and scales are as in Figure 2. Filled circles: first measurements; open circles: consecutive measurements; filled square: average values in healthy subjects (error bars: $\pm 1 \mathrm{SD}$ ).

mals, the VOR gain was higher when the head was accelerated toward the contralesional side, but at the subsequent velocity plateau horizontal gains were symmetric. The mathematical model by Lasker et al. accounts for these properties by implementing a linear and a nonlinear pathway on both sides. Figure 4 summarizes the main features of this model. 

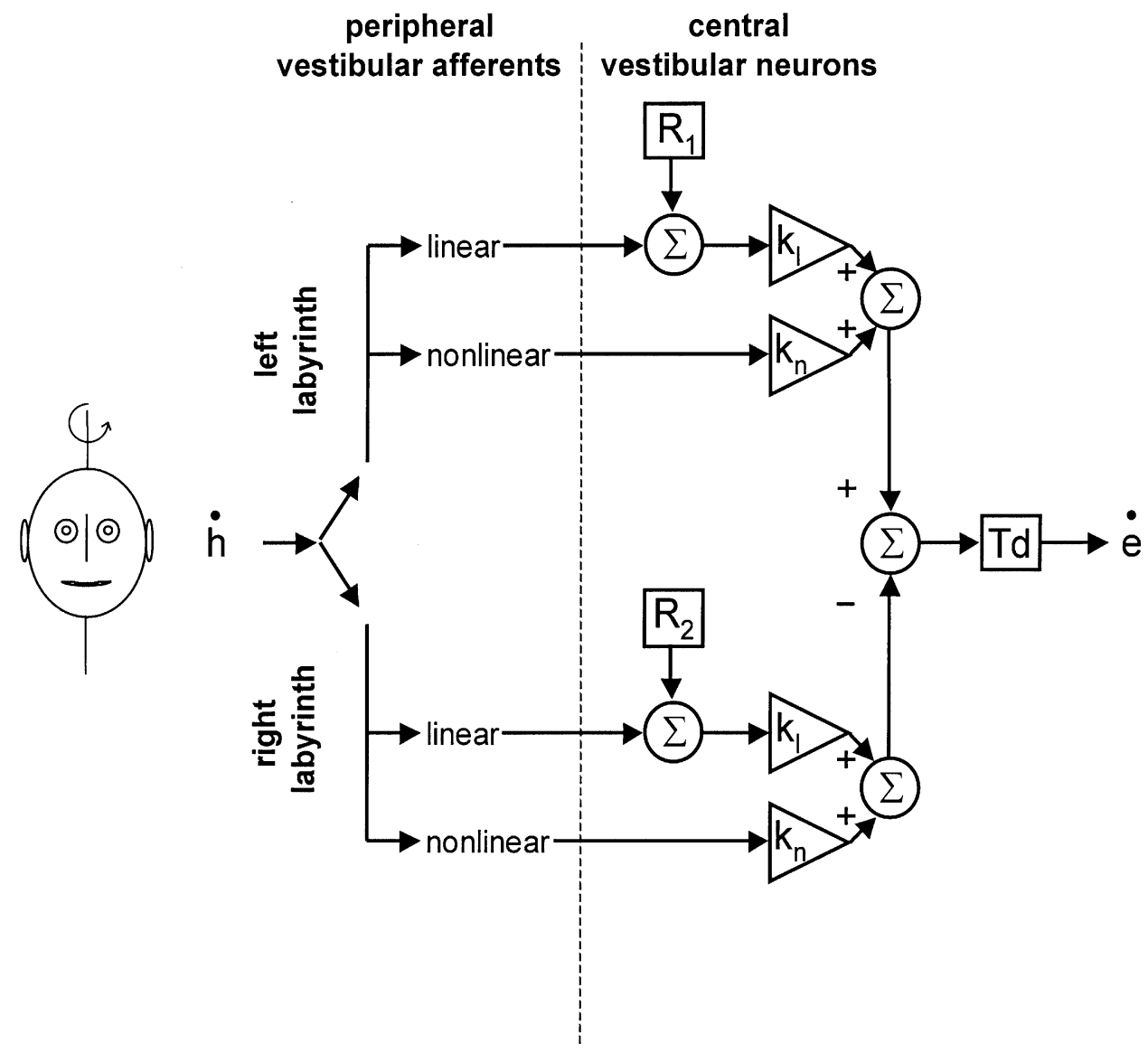

FIG. 4. Simplified diagram of the model described by Lasker et al. (2000). The input to the vestibuloocular reflex is angular head velocity $(\dot{h})$. This signal is passed through linear and nonlinear pathways. $k_{\mathrm{l}}$ and $k_{\mathrm{n}}$ represent the corresponding central gain elements of these two pathways. $R$ denotes the resting rate of central vestibular neurons, which is added to the linear pathway. $\mathrm{Td}$ : time delay of the reflex (set to $7 \mathrm{~ms}$ in our simulations).

The responses of the reflex during sinusoidal rotation with low peak velocities $\left(<30^{\circ} / \mathrm{s}\right)$ and during the velocity plateau after acceleration are attributed largely to the linear pathway that reacts relatively symmetrically upon labyrinthine inhibition and excitation. During sinusoidal rotation with higher frequencies and peak velocities, and during highacceleration steps, there is an additional but relatively small gain contribution from the ipsilateral nonlinear pathway. For instance, during a typical head impulse (duration $0.4 \mathrm{~s}$; amplitude $=40^{\circ}$; peak velocity $=$ $470^{\circ} / \mathrm{s}$; peak acceleration $=12,000^{\circ} / \mathrm{s}^{2}$ ), the gain contribution of the ipsilateral nonlinear pathway amounts to $11 \%$ according to the model. Therefore, a reduced vestibular signal from one labyrinth, e.g., after vestibular neuritis, will always result in an asymmetric gain during horizontal head impulses with the lower value during ipsilesional head rotation.

Lasker et al. (2000) found that in order to model the responses of hemilabyrinthectomized animals, they had to make the following adjustments in the model parameters: a decreased resting rate of ipsilateral central vestibular neurons $(R)$ by $11 \%$, an increased gain of the contralateral linear pathway $\left(k_{1}\right)$ by $20 \%$, and an increased gain of the contralateral nonlinear pathway $\left(k_{\mathrm{n}}\right)$ by $250 \%$. With these changes, which most likely are mediated by the central nervous system, the model does agree with the data obtained in the experimental animals. Note that in the context of the model, the term "gain" refers to a multiplication of an incoming signal, while for the behavioral description the VOR "gain" is defined by "eye velocity divided by head velocity".

The performance of the model upon stimulations used in our study is illustrated in Figure 5. A representative head impulse, as measured with a search coil placed on the forehead, served as input to the model (Fig. 5A). A right-sided unilateral vestibular deficit was simulated by increasing the deficit of the rightsided input (Fig. 5BD) from $0 \%$ (thin solid line corresponding to a normal right-sided vestibular input) to $100 \%$ (thick solid line corresponding to a total right-sided vestibular loss) with increments of $10 \%$ (dashed lines). Without central changes (Fig. 5B), no spontaneous nystagmus appears, and the unilateral deficit reduces the gain of the VOR not only during ipsilesional but also during contralesional head impulses. The gain is reduced bilaterally because a decrease of input into the linear pathway on the lesioned side affects the VOR gain on both sides equally. This symmetric effect is due to the fact that, during ipsilesional head impulses, the contralesional linear path- 

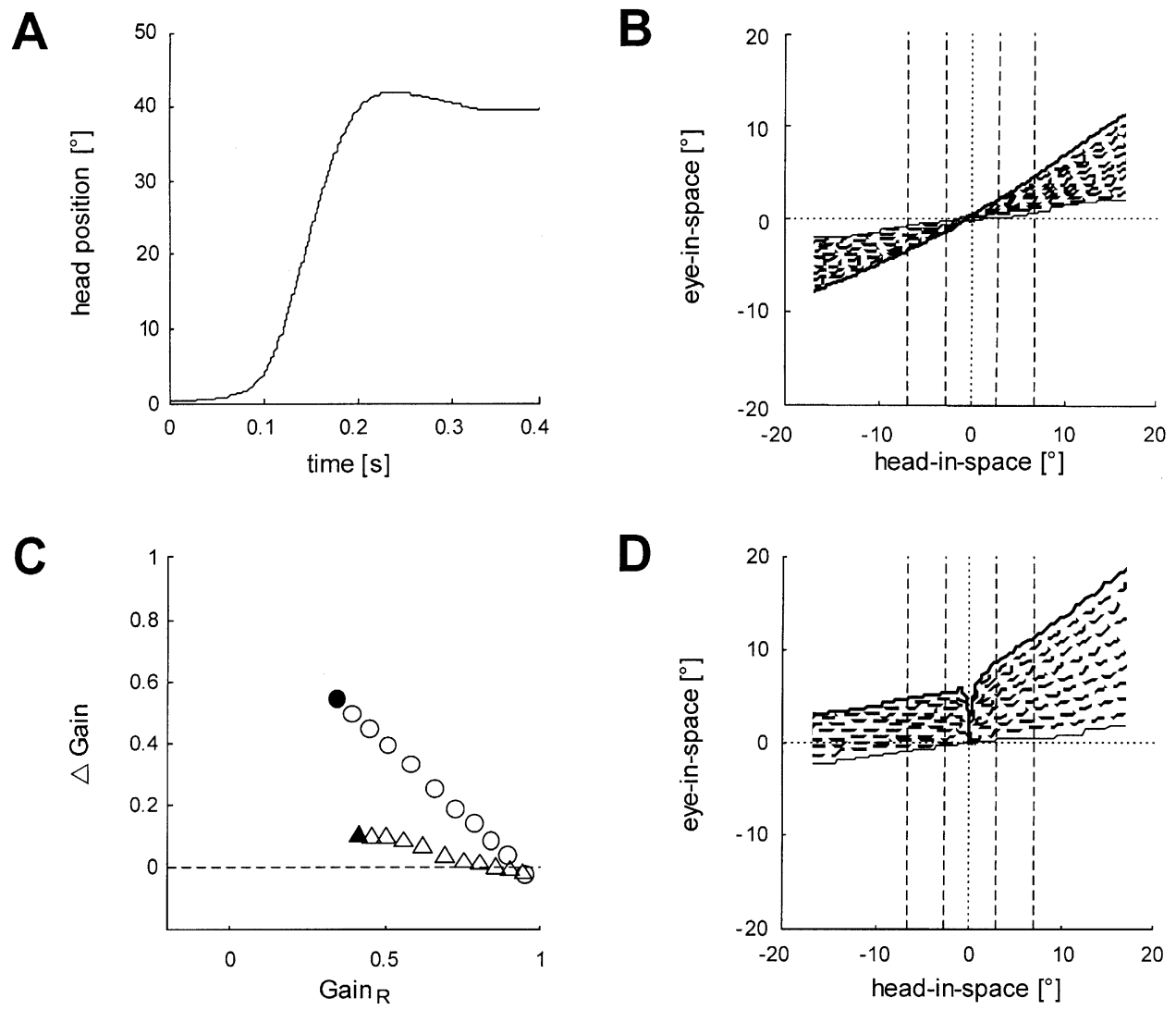

D

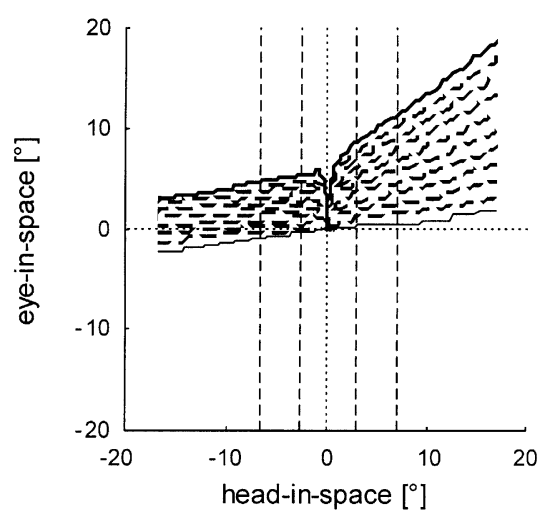

FIG. 5. Performance of the model by Lasker et al. (2000) upon an actual head impulse (applied by A.P.) as input (duration $=0.4 \mathrm{~s}$; amplitude $=40^{\circ} ;$ peak velocity $=470^{\circ} \%$ s; peak acceleration $=$ $12,000^{\circ} / \mathrm{s}^{2}$ ). A. Position trajectory of the head impulse. The small overshoot is typical. B. Eye-in-space vs. head-in-space in the presence of an increasing right-sided deficit. Each trace represents the VOR simulated by the model in the presence of a right-sided vestibular deficit. The function of the right labyrinth was reduced in 10 steps (dashed lines) from normal (no deficit, thin solid line) to total loss (no function, thick solid line). No central elements were changed. Dashed vertical lines indicate intervals for determining the gain

way is not driven into inhibitory cutoff. The computed small asymmetry between the bilaterally reduced VOR gains (gain lower during ipsilesional head rotation) is caused solely by the nonlinear pathway on the contralesional side, which is driven into inhibitory cutoff during ipsilesional head rotation but contributes to the gain during contralesional head rotation. Note that, in the absence of central changes, the impact of the contralesional nonlinear pathway on the VOR gain during contralesional head impulses is small. When central changes are permitted by changing ipsilesional $R(90 \rightarrow 80 \mathrm{~Hz})$, contralesional $k_{1}(1 \rightarrow 1.2)$, and contralesional $k_{\mathrm{n}}(0.00001 \rightarrow 0.000025)$ proportionally to the peripheral deficit (Fig. 5D), the model increases the VOR gain for contralesional head impulses and generates spontaneous nystagmus.

Plotting ipsilateral gain versus the difference between ipsi- and contralesional gain demonstrates the (see Methods). C. Gain difference ( $\Delta$ Gain) as a function of ipsilesional gain $\left(\right.$ Gain $\left._{R}\right)$. Data points represent the gains from panels $\mathbf{B}$ (triangles) and $\mathbf{D}$ (circles). Filled symbols: gains as a result of a total unilateral deficit. D. Axes and lines as in B, but ipsilateral $R(90 \rightarrow 80$ $\mathrm{Hz})$, contralateral $k_{\mathrm{l}}(1 \rightarrow 1.2)$, and contralateral $k_{\mathrm{n}}(0.00001 \rightarrow$ $0.000025)$ were changed proportionally to the peripheral vestibular deficit. Note that the spontaneous nystagmus manifests itself indirectly by eye-in-space displacements to the right evoked by the slightest head-in-space movement to either side, although it cannot be seen directly in this plot.

increase of gain difference as a function of the growing ipsilesional deficit (Fig. 5C). When ipsilesional $R$, contralesional $k_{1}$, and contralesional $k_{\mathrm{n}}$ remain unchanged, the increasing unilateral deficit has a small effect on gain asymmetry (triangle in Fig. 5C derived from Fig. 5B). In the presence of a total unilateral deficit, the gain difference amounts only approximately 0.1 (filled triangle). With simultaneous changes of all three variables, however, the asymmetry increases strongly as a function of the ipsilesional deficit (circles in Fig. 5C derived from Fig. 5D). A total unilateral deficit leads to a gain difference of about 0.55 (filled circle). Of course, with these simulations we do not suggest that central changes are in fact proportional to the unilateral peripheral vestibular deficit in individual patients. Other factors, such as time elapsed after the lesion, head movement activity, and age, will also play a role. 
Figure 6 compares the data of the patients with the prediction of the model. Again, gain differences are plotted as a function of the ipsilesional gain (compare with Fig. 5C). Based on the simulations, we identified an area that includes all possible output values resulting from variations of the unilateral peripheral deficit $(0 \%-100 \%)$ and of the parameters of central changes (ipsilesional $R$ and contralesional $k_{1}, k_{\mathrm{n}}$ ) proportional to the deficit. The data points of the patients showed a wide distribution: $61 \%$ were located inside the area that could be predicted by the model. The slope of the regression through all data points amounted to 0.76 and was approximately in between the simulations with (line a) and without (line b) changing ipsilesional $R$, contralesional $k_{1}$, and contralesional $k_{\mathrm{n}}$. Recall that the parameters of the model are derived from data obtained in experimental animals. Despite this caveat, the close approximation of the computer simulation with the majority of $\mathrm{VN}$ patients, in whom a purely unilateral deficit is suspected, is noteworthy.

\section{DISCUSSION}

Our study demonstrates that the asymmetry of the vestibulo-ocular reflex (VOR) evoked by horizontal head impulses decreases over time in patients after clinically suspected vestibular neuritis (VN). This improvement is due mainly to a gain increase for head impulses toward the ipsilesional side and takes place primarily within the first few weeks after the onset. Our results are in contrast to the findings in patients after unilateral neurectomy, in whom the gain asymmetry remained unchanged over time (Halmagyi et al. 1990). The same lack of improvement of the horizontal high-acceleration VOR was also observed in guinea pigs with complete unilateral vestibular loss (Vibert et al. 1993; Gilchrist et al. 1998). Thus, it is fair to assume that the observed ipsilesional VOR gain recovery is a result of spared peripheral vestibular function on this side. Whether the ipsilesional gain increase is a result of incremental recovery of peripheral vestibular function or whether unchanged residual peripheral input drives an emerging central compensation mechanism cannot be inferred from our results.

How does the gain for the contralesional head impulse relate to the ipsilesional deficit? To answer this question, we need to consider the physiology of central vestibulo-ocular pathways. The model developed by Lasker et al. (2000) on the bases of experimental results in squirrel monkeys (see Fig. 4) makes an interesting prediction for unilateral peripheral vestibular deficits: Without central adaptation leading to internal gain increases of the contralesional linear and non- linear pathways and a decrease of the resting rate of the ipsilesional central vestibular neurons, the gain for contralesional head impulses declines much more than was observed in the majority of our patients and in the patients after unilateral vestibular neurectomy reported by others (Halmagyi et al. 1990; Aw et al. 1996, 2001). According to the model by Lasker et al., a total unilateral vestibular deficit results, without adjustments of the central internal gains and the resting rate, in an ipsilesional VOR gain of $\sim 0.4$ and a contralesional VOR gain of $\sim 0.5$ (filled triangle in Fig. $5 \mathrm{C}$ ). On the other hand, effective adaptive changes of the central internal gains and the resting rate lead to an ipsilesional VOR gain of $\sim 0.3$ and a contralesional VOR gain of $\sim 0.9$ (filled circle in Fig. $5 \mathrm{C}$ ).

The output of the model by Lasker et al. (2000) can be qualitatively explained by considering the symmetric and asymmetric properties of central vestibular pathways. The linear pathways contribute equally during ipsilateral and contralateral rotations, even during high accelerations. In other words, a unilateral deficit of the linear pathways leads to the same gain reduction on both sides. In the presence of a unilateral vestibular deficit, the higher gain on the contralesional side is a result of the adapted contralesional nonlinear pathway that enhances the VOR mainly during contralesional head impulses. Recall that during ipsilateral head impulses, the contralesional nonlinear pathway is driven into inhibitory cutoff. In the absence of central changes, the intact contralesional nonlinear pathway contributes little to the contralesional gain. With the central changes implemented in the model, however, the contribution of the adapted nonlinear pathway becomes large.

Ewald's second law states that excitation drives the vestibulo-ocular reflex better than inhibition (Ewald 1892). In the model of Lasker et al., Ewald's second law is implemented by the nonlinear pathway that is driven mainly by excitatory semicircular canal input. While in healthy subjects the impact of the nonlinear pathway, and hence Ewald's second law, is small, the situation is different after a unilateral decrease of peripheral vestibular function. In this case, the model assumes central adaptive changes of the nonlinear pathway to keep the contralesional VOR gain close to normal, i.e., Ewald's second law becomes more powerful.

According to the model, the more effective central compensation is, the closer the contralesional gains move up to the normal range. For that reason, assuming one applies the model by Lasker et al., the relatively small reduction of contralesional gains, as observed in the majority of our patients, can be explained only by central "upregulation". In the model this is achieved by increasing the gains of contralesional linear and nonlinear vestibulo-ocular pathways. Based on these considerations, a small contralesional 


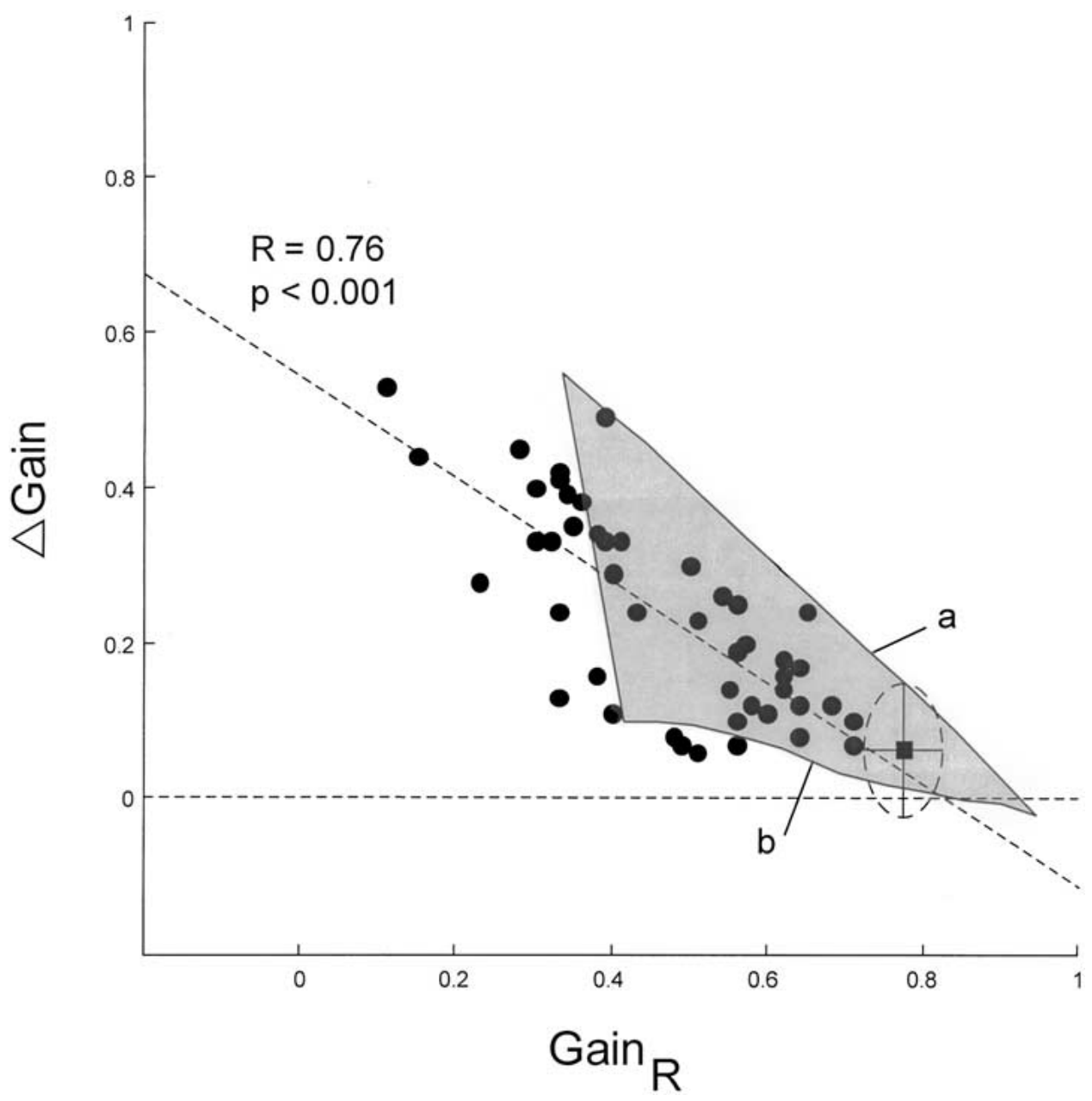

FIG. 6. Comparison of patients' data with that from the model by Lasker et al. (2000) $\Delta$ Gain: differences between gains; Gain ${ }_{R}$ : gains during head impulses toward the right side, which was always the weaker side. Filled circles: individual testing sessions $(N=47$ in 37 patients); filled square: average data point from healthy subjects (ellipse with horizontal and vertical radii: $\pm 1 \mathrm{SD}$ ); horizontal dashed

gain reduction of high-acceleration VOR in a unilateral peripheral deficit should not be interpreted as central "downregulation" that the brain imposes to decrease the asymmetry of the horizontal VOR (Fetter and Zee 1988). A small asymmetry, rather, speaks for a deficient central "upregulation" of the contralesional gain. Indeed, it seems that the central nervous system does not attempt to balance the horizontal VOR gains but instead tries to boost the contralesional gain to a value close to normal.

Judged by the weak correlation between ipsilesional gain and the difference of VOR gain between right and left, the effectiveness of this centrally mediated contralesional upregulation seems to vary considerably among patients with VN (Fig. 6). Moreover, patients with minimal upregulation seem to closely resemble patients with a symmetrical bilateral peripheral deficit. This makes it difficult to de- line: $\Delta$ Gain $=0$; oblique dashed line: linear regression through data cloud. Gray area: all possible output values of the model when one varies the unilateral peripheral deficit (0-100) and gradually changes the central elements in proportion to the deficit (see Fig. 4). Line a: full changes of central elements (corresponding to Fig. 5D). Line b: no changes of central elements (corresponding to Fig. 5B).

cide in these cases whether a unilateral deficit with insufficient upregulation or a bilateral deficit is present. If gains are outside of the area explainable by a total unilateral peripheral vestibular deficit, a bilateral lesion should be considered (Fig. 6, left of the gray area). In fact, more than one third of our patients had initial gains that suggested bilateral VN, although the gains were asymmetrical. However, agedependent parameters, which presently are not included in the model, could shift the leftmost border of the area further to the left so that the percentage of bilaterally affected patients would be lower. Also, we point out that the computer simulations relied on the assumption that the primary afferents of linear and nonlinear pathways are equally affected by $\mathrm{VN}$. Finally, we reemphasize the caveat that the parameters of the model are based on experiments in squirrel monkeys. 
Of course, we were not able to separate unilateral from bilateral cases in vivo. However, postmortem findings in unselected human preparations of bilateral infections of the labyrinth or the vestibular ganglion by herpes simplex virus type 1 in the majority of cases (Arbusow et al. 2001), supports the hypothesis that at least some of our patients after $\mathrm{VN}$ were affected bilaterally. Future studies should try to correlate data from vestibular testing with data from methods that allow quantifying inflammations of the vestibular nerve and labyrinth on both sides, e.g., by MRI (Karlberg et al. 2004).

In conclusion, we have demonstrated that, after vestibular neuritis, the asymmetry of horizontal gain of high-acceleration VOR decreases during the first few weeks due to an ipsilesional gain increase. We conjecture that the main source of the gain asymmetry is due to a central upregulation of the contralesional gain, which is already present during the first measurements after the onset of the vestibular neuritis, In addition, we have provided indirect evidence that a number of patients, after a sudden peripheral vestibular asymmetry, may suffer from asymmetrical bilateral vestibular neuritis.

\section{ACKNOWLEDGMENTS}

The authors thank Dr. D.M. Lasker for valuable comments; Dr. A. Schmid-Priscoveanu for performing some of the earlier measurements; and H. Obzina, S. Bizzarri, and T. Schmückle for technical assistance. This work was supported by the Swiss National Science Foundation (32-51938.97 SCORE A / 31-63465.00) and the Betty and David Koetser Foundation for Brain Research, Zurich, Switzerland.

\section{REFERENCES}

Allum JH, Ledin T. Recovery of vestibulo-ocular reflex-function in subjects with an acute unilateral peripheral vestibular deficit. J. Vestib. Res. 9:135-144, 1999.

Arbusow V, Schulz P, Strupp M, Dieterich M, von ReinhardstoettNer A, Rauch E, Brandt T. Distribution of herpes simplex virus type 1 in human geniculate and vestibular ganglia: implications for vestibular neuritis. Ann. Neurol 46:416-419, 1999.

Arbusow V, Strupp M, Wasicky R, Horn AK, Schulz P, Brandt T. Detection of herpes simplex virus type 1 in human vestibular nuclei. Neurology 55:880-882, 2000.

Arbusow V, Theil D, Strupp M, Mascolo A, Brandt T. HSV-1 not only in human vestibular ganglia but also in the vestibular labyrinth. Audiol. Neurootol. 6:259-262, 2001.

Aw ST, Halmagy GM, Haslwanter T, Curthoys IS, Yavor RA, Todd MJ. Three-dimensional vector analysis of the human vestibuloocular reflex in response to high-acceleration head rotations. II. responses in subjects with unilateral vestibular loss and selective semicircular canal occlusion. J. Neurophysiol. 76:4021-4030, 1996.

Aw ST, Fetter M, Cremer PD, Karlberg M, Halmagy GM. Individual semicircular canal function in superior and inferior vestibular neuritis. Neurology 57:768-774, 2001.

BRANDT T. Vestibular neuritisn Vertigo: Its Multisensory Syndromes. Springer-Verlag, London, pp 67-79, 2001.

Brantberg K, Magnusson M. The dynamics of the vestibulo-ocular reflex in patients with vestibular neuritis. Am. J. Otolaryngol. 11:345-351, 1990.

Ewald EJ. Physiologische Untersuchungen über das Endorgan des Nervus Octavus. Bergmann, Wiesbaden, 1892.

Fetter M, Zee DS. Recovery from unilateral labyrinthectomy in rhesus monkey. J. Neurophysiol. 59:370-393, 1988.

Furuta Y, Takasu T, Fukuda S, Inuyama Y, Sato KC, Nagashma K. Latent herpes simplex virus type 1 in human vestibular ganglia. Acta Otolaryngol. Suppl. 503:85-89, 1993.

Gilchrist DP, Curthoys IS, CARTwright AD, Burgess AM, Topple AN, Halmagy M. High acceleration impulsive rotations reveal severe long-term deficits of the horizontal vestibulo-ocular reflex in the guinea pig. Exp. Brain Res. 123:242-254, 1998.

Halmagy GM, Curthoys IS. A clinical sign of canal paresis. Arch. Neurol. 45:737-739, 1988.

Halmagy GM, Curthoys IS, Cremer PD, Henderson CJ, Todd MJ, STAPLes MJ, D'Cruz DM. The human horizontal vestibulo-ocular reflex in response to high-acceleration stimulation before and after unilateral vestibular neurectomy. Exp. Brain Res. 81:479490, 1990.

Imate Y, Sekitani T. Vestibular compensation in vestibular neuronitis. Long-term follow-up evaluation. Acta Otolaryngol. 113:463-465, 1993.

Karlberg M, Annertz M, Magnusson M. Acute vestibular neuritis visualized by 3-T magnetic resonance imaging with high-dose gadolinium. Arch. Otolaryngol. Head Neck Surg. 130:229-232, 2004.

LASKer DM, Hullar TE, Minor LB. Horizontal vestibuloocular reflex evoked by high-acceleration rotations in the squirrel monkey, III. Responses after labyrinthectomy. J. Neurophysiol. 83:2482-2496, 2000.

PAIGE GD. Nonlinearity and asymmetry in the human vestibuloocular reflex. Acta Otolaryngol. 108:1-8, 1989.

Schmid-Priscoveanu A, Straumann D, Bohmer A, Obzina H. Vestibulo-ocular responses during static head roll and three-dimensional head impulses after vestibular neuritis. Acta Otolaryngol. 119:750-757, 1999.

Schmid-Priscoveanu A, Bohmer A, Obzina H, Straumann D. Caloric and search-coil head-impulse testing in patients after vestibular neuritis. J. Assoc. Res. Otolaryngol. 2:72-78, 2001.

Schuknecht HF, Kitamura K. Second Louis H. Clerf Lecture. Vestibular neuritis. Ann. Otol. Rhinol. Laryngol. Suppl. 90:1-19, 1981.

Schulz P, Arbusow V, Strupp M, Dieterich M, Rauch E, Brandt T. Highly variable distribution of HSV-1-specific DNA in human geniculate, vestibular and spiral ganglia. Neurosci. Lett 252:139-142, 1998.

Straumann D, Zee DS. Three-dimensional aspects of eye movements. Curr. Opin. 8:69-71, 1995.

Vibert N, de Waele C, Escudero M, Vidal PP. The horizontal vestibulo ocular reflex in the hemilabyrinthectomized guinea-pig. Exp. Brain Res. 97:263-273, 1993. 